\title{
Fast detection of pathogenic bacteria by using different sensor techniques
}

\author{
K. Kleo ${ }^{1}$, C.Nietzold ${ }^{1}$, R. Grunow ${ }^{2}$, F. Lisdat ${ }^{1}$ \\ ${ }^{1}$ University of Applied Sciences Wildau, Biosystems Technology, \\ Bahnhofstrasse 1, 15745 Wildau, Germany \\ flisdat@th-wildau.de \\ ${ }^{2}$ Centre for Biological Security (ZBS2), \\ Robert Koch Institute, Nordufer 20, 13353 Berlin, Germany
}

\begin{abstract}
:
Francisella tularensis are gram-negative bacteria capable of infecting mammals. This high pathogenic species is classified as a potential bioterroristic agent by the Centers for Disease Control and Prevention, Atlanta / USA [1]. Therefore, a renewed interest in studying F.tularensis bacteria can be observed. This is strongly focused on developments of fast, cheap, robust, sensitive and specific detection methods. With this work we want to present alternative sensor systems for these pathogenic bacteria.

Here we demonstrate two different immunological detection systems for F.tularensis. First technique is based on quartz crystal microbalance (QCM) which uses sensor chips modified by a specific antibody. The QCM technique is combined with a microfluidic flow system and allows the online detection of binding events between antibody and whole bacteria. The second system is based on the aggregation of antibody-functionalized gold nanoparticles and bacteria. This is monitored by a change of particle diameter using dynamic light scattering and of absorption peak shift by UV/Vis spectroscopy. A detection limit of $10^{3} \mathrm{CFU} / \mathrm{ml}$ can be obtained.
\end{abstract}

Key words: quartz crystal microbalance (QCM), gold nanoparticles, bacteria detection, signal enhancement, Francisella tularensis

\section{Introduction}

To identify Francisella tularensis bacteria histological sections [02] and also cell culture or bio-molecular techniques [03] are used. These standard techniques are sensitive and useful for analysis. However these techniques are rather time consuming for a rapid identification of Francisella tularensis which is required in a bioterroristic context. Additional techniques are needed to provide a fast detection and identification of this pathogenic species. Biosensors are the fastest growing technology for pathogen detection compared to other technologies [04] and can manage this task in principle. Quartz crystal microbalance (QCM), can be used as a mass sensitive device for the detection of binding events by measuring the change in frequency of a quartz resonator [05]. It allows the evaluation and characterization of surface modifications, adsorption processes and also interaction of biomolecules. The integration of nanotechnology approaches in the field of sensor development holds great promise [06]. In this context, nanoparticles
(NPs) have raised great expectations with respect to reducing response times and using them in multiplexed systems. Here we demonstrate two different immunological detection systems for Francisella tularensis bacteria (figure 1).

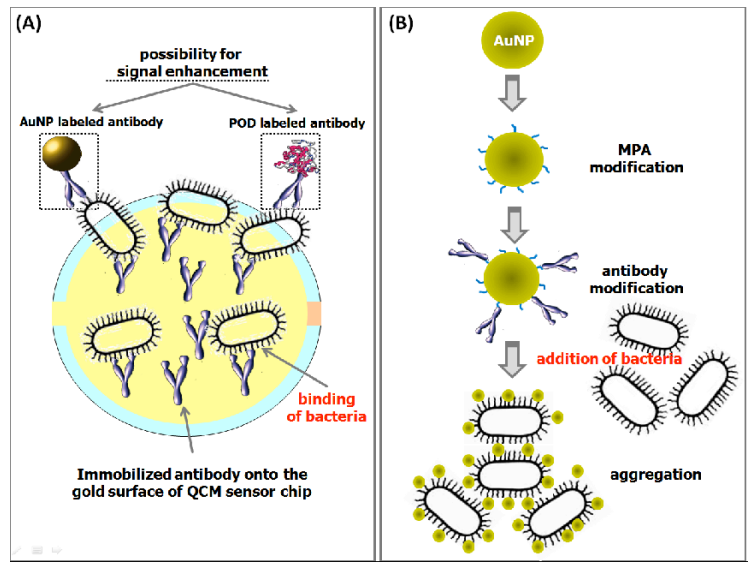

Fig. 1. Model of sensor systems used for fast bacteria detection. Figure illustrated the sensorprinciple of the quartz crystal microbalance technique (A) and the formation of aggregates by using antibody-functionalized gold nanoparticles (B). 


\section{First Sensor Model}

The quartz crystal microbalance (QCM) belongs to the category of bulk acoustic wave devices and can be used as mass sensitive biosensor. This technique allows a fast immunological detection of F.tularensis bacteria. The principle is based on QCM sensor chips modified by a specific antibody. These antibodies function as a capture molecule directed against the lipopolysaccharide (LPS) structure on the surface of bacteria cell wall. By combining QCM with a microfluidic flow system an online detection of binding events between antibody and whole bacteria is possible. The mass attachment results in a defined shift of frequency signals and a detection limit of about $10^{3} \mathrm{CFU} / \mathrm{ml}$ can be obtained (see figure $2 \mathrm{~A}$ ). It can be also shown that the reaction is high specific (see figure 2 B). Furthermore we demonstrate two possibilities for specific and significant signal enhancement by using antibody functionalized gold nanoparticle or a second peroxidase-labeled antibody and substrate conversion (see figure 3 ). These additional steps can be seen as a further proof of specificity.

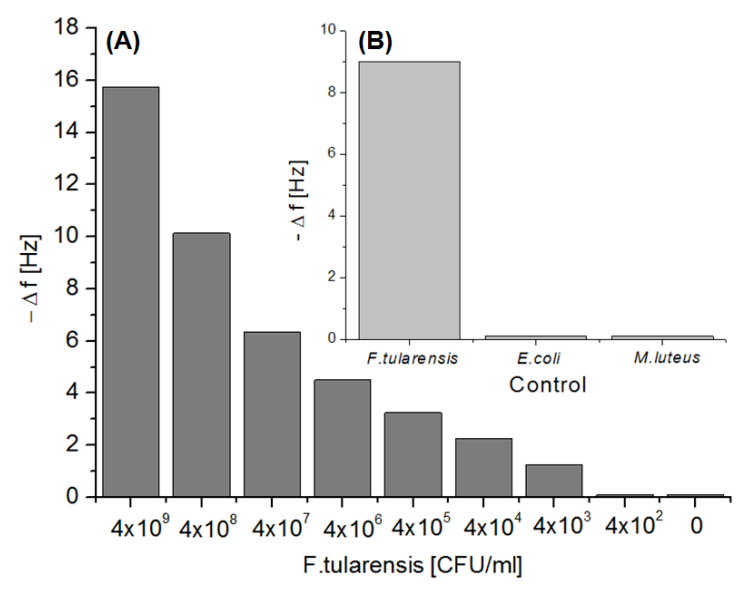

Fig. 2. (A) Detection limit of Francisella tularensis bacteria in QCM measurements. Shown is the frequency shift. (B) Control measurement using Francisella tularensis antibody to detect $10^{8} \mathrm{CFU} / \mathrm{ml}$ of F.tularensis, E.coli and M.luteus bacteria.

\section{Second Sensor Model}

The second immunological detection system is based on the aggregation of antibodyfunctionalized gold nanoparticles with bacteria. Gold nanoparticles are characterised by their intensive light absorption which is caused by excitation of collective oscillations of valence band electrons, called plasmon resonance. The plasmon resonance wavelength is, amongst others, a function of the direct particle environment so that changes therein can be monitored spectroscopically. For bacteria

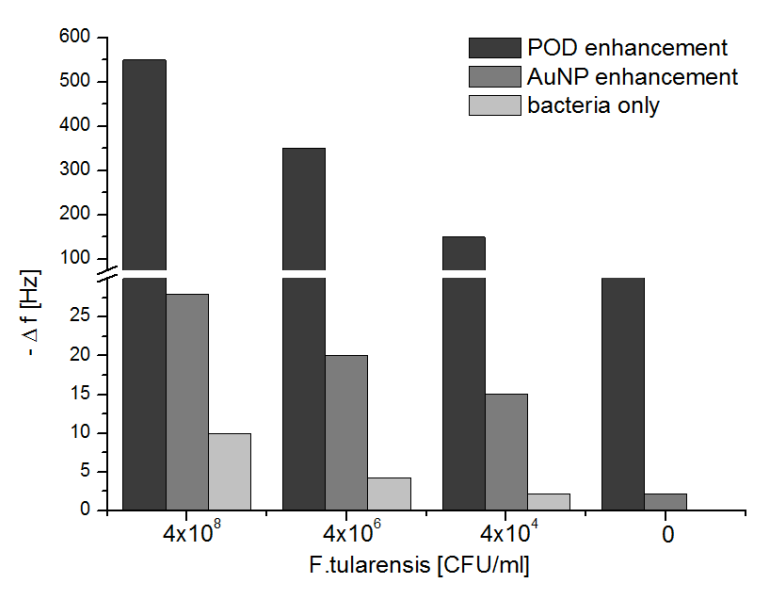

Fig. 3. The figure shows absolute frequency changes for bacteria binding, Au-NP-antibody binding and precipitate formation by using $P O D$ labeled antibodies for different concentrations of F.tularensis bacteria. The increased frequency change after signal enhancement strategies is caused by additional mass of Au-NP or precipitating substrates by peroxidase conversion.

For bacteria detection the surface modification, functionalization and bioconjugation of gold nanoparticles (size $10 \mathrm{~nm}$ ) is investigated. The test system based on a simple mixing of the bacterial sample with functionalized Au-NP solution in a volume ratio $1: 1$ and evaluates the optical changes (light scattering, absorption). After anti-F.tularensis immobilisation it is also possible to quantify an increase of about $10 \mathrm{~nm}$ in relation to the MPA-modified gold nanoparticles via DLS measurements verifying the successful fixation of an antibody layer on the Au-NP. Binding of F.tularensis to antibodyfunctionalized gold nanoparticles and subsequent complex formation is first confirmed by DLS measurements. A clear increase in the mean particle diameter can be detected after the immune reaction. Sensitivity can be here provided in the concentration range $4 * 10^{5}-$ $4 * 10^{8} \mathrm{CFU} / \mathrm{ml}$ (figure 4).

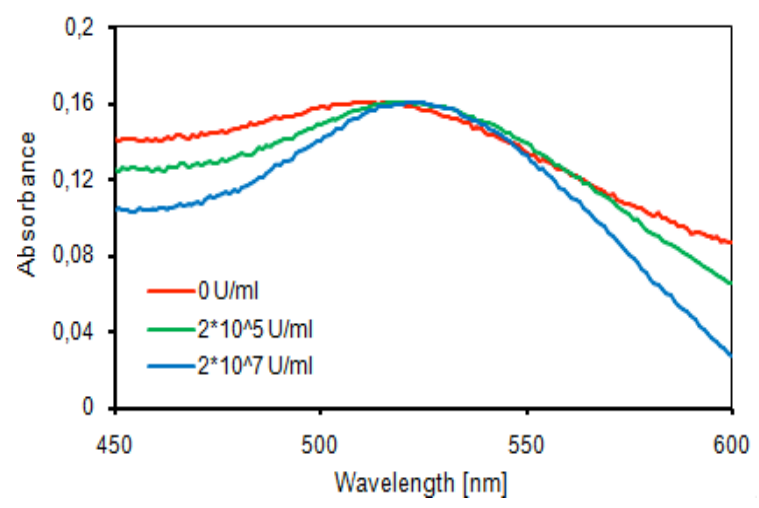

Fig. 4. UV/Vis spectra of Au-NP solutions after preparation and F.tularensis detection: Antibodyfunctionalized gold nanoparticles (red), after addition of $4 \times 10^{5} \mathrm{CFU} / \mathrm{ml}$ F.tularensis (green), after addition of $4 \times 10^{7} \mathrm{CFU} / \mathrm{ml}$ F.tularensis (blue). 
Using UV/Vis spectroscopy the functionalized nanoparticles have an absorbance maximum at $515 \mathrm{~nm}$ that increases to $524 \mathrm{~nm}$ following aggregation as shown in figure 5 . Both methods indicate that the bacteria are recognized onto the surface of the antibody-functionalized gold nanoparticles resulting in complex formation.

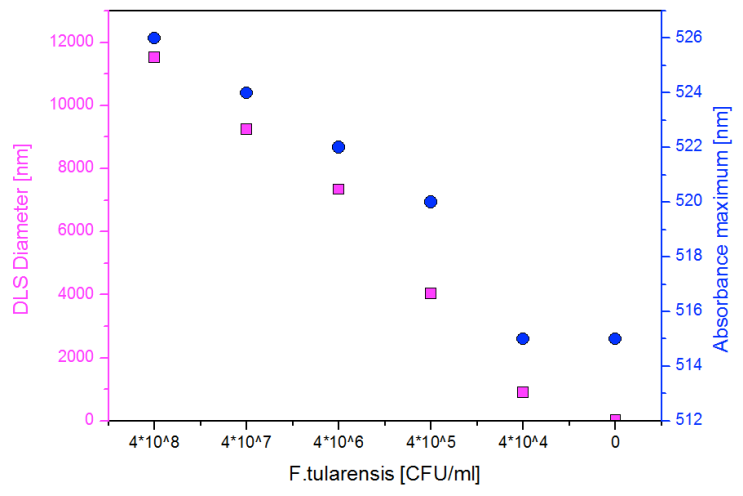

Fig. 5. DLS and UVNis data of $10 \mathrm{~nm}$ gold nanoparticle after addition of different concentrations of F.tularensis.

\section{Conclusion}

This work demonstrates a non-labeled detection system for direct identification of inactivated Francisella tularensis bacteria by using two different sensor strategies. By using quartz crystal microbalance combined with a microfluidic flow system a detection limit up to $4 \times 10^{3} \mathrm{CFU} / \mathrm{ml}$ can be observed. A fast sensor response after $20 \mathrm{~min}$ allows rapid reaction of the first responder. Furthermore two strategies for signal enhancement using modified antibodies are useful techniques for further specification analysis.

A second sensor principle based on bioconjugated gold nanoparticles through the formation of bacteria complexes interconnected by Au-NP. Sensitivity can be provided in the concentration range of $4 \times 10^{5}-4 \times 10^{8} \mathrm{CFU} / \mathrm{ml}$ for F.tularensis analysis without any further amplification. The results demonstrate that DLS and UV/Vis spectroscopy of Au-NP solution can be a promising analytical tool for determination of F.tularensis.

Both presented immunodetection systems have shown attractive advantages with respect to speed and simplicity for pathogenic bacteria analysis. A next challenged would be the investigation of matrices and other binding molecules to be close to a bioterrorism scenario.

\section{Acknowledgments}

The authors would like to acknowledge funding by the BMBF-Gemany via the BioNapaTest project (PNT51513) and BigRudi project (13N9593). Furthermore we acknowledge the cooperation with the Centre for Biological Security of RobertKoch Institute (Berlin, GER) for Francisella tularensis bacteria sample preparation.

\section{References}

[1] Bioterrorism Overview, Centers for Disease Control and Prevention, 2008-02-12, http://www.bt.cdc.gov/bioterrorism/overview.asp, retrieved 2011-012-08

[2] K.A. Karlsson, S. Dahlstrand, E. Hanko \& O. Soderind (1970). Demonstration of Francisella tularensis in sylvan animals with the aid of fluourescent antibodies. Acta Pathol. Microbiol. Immunol. Scand. (B), 78, 647-651.

[3] H. Tomaso, H.C. Scholz, H. Neubauer, A.L. Dahouk, E. Seibold, O. Landt, M. Forsman \& W.D.Splettstoesser W.D. (2007). Real-time PCR using hybridization probes for the rapid specific identification of Francisella tularenis subspecies tularensis. Mol. Cell. Probes, 21 (1), 12-16.

[4] O. Lazcka, F.J. Del Campo \& F.X. Munoz, Pathogen detection: a perspective of traditional methods and biosensors. Biosens Bioelectron, 2007. 22(7): p. 1205-17.

[5] Marx, K.A., Quartz crystal microbalance: a useful tool for studying thin polymer films and complex biomolecular systems at the solution-surface interface. Biomacromolecules, 2003. 4(5): p. 1099-120.

[6] N. Sanvicens, M.P. Marco, Multifunctional nanoparticles--properties and prospects for their use in human medicine. Trends Biotechnol. 2008 Aug;26(8):425-33. 